\title{
An Approach of an Image Watermarking Scheme using Neural Network
}

\author{
Asmaa Qasim Shareef, Ph.D \\ Science College $\backslash$ Computer Science Dep. \\ University of Baghdad
}

\author{
Roaa Essam Fadel \\ Science College $\backslash$ Computer Science Dep. \\ University of Baghdad
}

\begin{abstract}
An approach of an image watermarking scheme using neural network is presented in this work. In the proposed approach, watermark comes from the weights of an identify image that are loaded from a learned feed-forward neural network, the neural network is learned by using the back-propagation learning algorithm. To improve the robustness of watermarked image; the procedure of watermark embedding is embedded into host image through selecting and modifying the Gaussian coefficients comes from a noisy image. The noised image is damaged by the salt and pepper noise. In order to identify the cover of extracted watermark, feedforward neural network is used in the watermarking identification to overcome the limitation of unknown data comes randomly. The results of the scheme realization show the robustness of proposed scheme that has preferable performance for both identification and watermarking of a noised image
\end{abstract}

\section{General Terms}

Watermarking, Back-propagation algorithm, Identification

\section{Keywords}

feed-forward neural network, salt and pepper noise, Gaussian filter coefficients.

\section{INTRODUCTION}

For online shops by customer identification watermarking digital watermarking is a technique for digital multimedia copyright protection, today it is used in various applications offering an alternative to more restrictive digital rights management approaches. The main requirements of the watermark are the robustness and the imperceptibility. Image is considered as a communication channel to transmit messages in the watermarking schemes. Different image has different watermarking capacity, therefore; watermarking capacity should be associated with the content of image [1]. Watermark allows invisible mark to be placed on top of normal information, so it is an important protection and identification technique. Normal information such as bank note, ID card, magnetic card and valuable documents. When visibility to users is required, watermarking techniques is developed to protect digital images against illegal reproduction and illegal modifications. A simple and widely used technique for watermarking images is to add a pattern on top of an existing image. Usually this pattern is an image itself a logo or something similar, which distorts the underlying image. In Fig.(1), the pattern is the red middle image while the portrait picture of man's face is the image being watermarked [2].
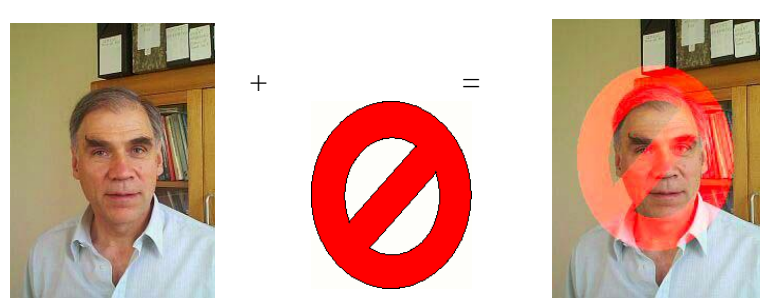

Fig. (1) Visible watermarking [2]

It is possible to reverse any, i.e. In a standard image editor it is possible to merge both images and get a watermarked image.

Adverse effects so that the original doesn't need to be kept. This method is only really applicable to watermarking, as the pattern is visible and even without the original watermark, it is possible to remove the pattern from the watermarked image with some effort and skill.

This paper, offers an approach based on back-propagation Neural Network [3] to train a given cover image to produce a desired watermark image. The approach for hiding the weights into the cover image has been designed in such a way that it does not produce visual deterioration of the original cover image. This method is extra secure as it leads to watermarking indirectly the noised image with the salt and pepper: the Gaussian filter [4] has been established for restoration of images contaminated by the noise as reliable method to remove it without damaging the edge details, the weights result were uploaded into the multilayer feed-forward neural network to produce the enhanced image which turn to be the watermarked image into the cover. At the end of the training, the entire trained neural network weights have been hidden within the cover image itself.

The organization of the paper is as follow: Section 2 describes the literature survey of watermarking algorithms, how to process the image noised with salt and pepper, and emphasizes on neural network watermarking algorithm. Section3 gives algorithms about visible watermark embedded in noisy images. Section 4 analyzes the results. Conclusion is discussed in section5. The paper ends with the references.

\section{LITERATURE SURVEY}

The current literature is rich in watermarking algorithms developed for various types of media, such as image, and text data. The algorithms work in various domains like spatial, DCT, DWT, wavelet, and neural also implemented on FPGA for real time watermarking. For images corrupted by salt and pepper noise and the use of neural network in watermarking. [5] Provide an introduction to three strategies to support fast 
watermark embedding: Container watermarking based on precalculations which today are already used in industrial applications, client-server watermarking where the content is marked after the transfer to the customer and grid watermarking using the computational power of grid networks. [6] Discuss the feasibility of coding an "undetectable" digital water mark on a standard $512 * 512$ intensity image with an 8 bit gray scale. Two methods of implementation are discussed. The first is based on bit plane manipulation of the LSB, which offers easy and rapid decoding. The second method utilizes linear addition of the water mark to the image data, and is more difficult to decode, offering inherent security. [7] Present a new adaptive watermarking algorithm. The adaptive watermark is generated from the image content and can be treated as a digital fingerprint of the image, it is robust against any degradation, hacking and attack. [8] Propose algorithm combines advantages of both wavelet based frequency domain watermarking which provides the robustness against different attacks and bit plane slicing scheme of spatial domain watermarking which provides lesser computational complexity suitable for real time implementation that was implemented on FPGA. [9] Propose a method based on the kFill algorithm that can be accomplished in single-pass scan over the image. The algorithm is capable to remove simultaneously both salt noise and pepper noise of any sizes that are smaller than the size of document objects. A modified decision based unsymmetrical trimmed median filter algorithm for the restoration of gray scale, and color images that are highly corrupted by salt and pepper noise is proposed in [10]. [11] Propose a two-phase scheme for removing saltand-pepper impulse noise. In the first phase, an adaptive median filter is used to identify pixels which are likely to be contaminated by noise (noise candidates). In the second phase, the image is restored using a specialized regularization method that applies only to those selected noise candidates. [12] Propose a DWT domain image watermarking; watermark comes from a binary image encrypted by two-dimensional chaotic stream encryption. The watermark is embedded into host image through selecting and modifying the wavelet coefficients using Genetic algorithm In order to identify the owner of extracted watermark, Synergetic Neural Network is used in the watermarking identification to overcome the limitation of correlation analysis or the human sense organ after some attacks. [13] Offer a technique based on backpropagation Neural Network to train a given cover image to produce a desired watermark image. At the end of the training, the entire trained neural network weights to be hidden within the cover image itself. By extraction techniques, the weights can be derived from the cover image and used to reconstruct the trained Neural Network again which in turn converts the cover image into desired watermark image. [14] Used multi-layer feed-forward Neural Network to model human visual system and present an imageadaptive method of watermarking for the watermarking on DCT coefficients. In [15] an image watermarking approach based on the human visual system model and neural network technique is proposed as an attempts to define an adaptive watermarking scheme based on Multi-Layer feed-forward neural networks to determine the strength of watermark bit that should be embedded into the middle-frequency coefficients of block DCT domain of the host image.

\section{ALGORITHMS}

\subsection{Gaussian Filter}

Read the noised image with salt and pepper, which is represented in Fig.(4-a), put the value of the image intensity into a matrix with size of $w^{*} h$, (e.g 250*250) where "w" represent the image width and the " $h$ " represent the image height.

The process of learning the neural network about the noised image will take two steps:

First step: Apply the Gaussian law to remove the noise; this operation will take the following steps:

a) Apply window slide operation on the image matrix to divide it in to $\mathrm{N}^{*} \mathrm{~N}$ window (e.g. $3 * 3$ )

b) Find the value of the fuzzy function " $u\left(g_{s t}\right)$ " for all the neighbors pixels of the center pixel of the window " $g_{s t}$ " this step represent a fuzzy process to the pixels of the noisy image and represent as the equation below:

$$
u\left(g_{s t}\right)=\exp \left[\frac{-\left(g_{c}-g_{s t}\right)^{2}}{\alpha}\right] \exp \left[\frac{-d^{2}}{\beta}\right]
$$

c) Find the new value of the center pixel and all its neighbors' pixels inside the window as follow:

$$
g_{s t}^{\prime}=u_{s t} g_{s t}
$$

d) Rearrange all the new values of the pixel in the window and then take the medium value and replace it with the center value of the window:

$$
f_{i j=\text { medium }}\left\{g_{s t}^{\prime}\right\} \quad \text { where } s, t \in i, j
$$

Second step: The output image that is shown in

Fig.(4-b), will be entered through back-

propagation learning algorithm in order to learn

the multi-layered feed-forward neural network

after applying the window slide to split the image

into wind of $3 * 3$ and enter each row of this window

to learn it and save output in anew array and the

weights in file.

\subsection{Multi-layered Feed-Forward Neural Network}

(i) Neurons' no. of inputs in the input layer is 3 .

(j) Neurons' no. of inputs in the hidden layer is 3 .

(k)Neurons' no. of inputs in the output layer is 3 .

- Initialize randomly by $(-0.5,0.5)$ both the weights connecting the input layer to the hidden layer, W1 $\left(\mathrm{w}_{\mathrm{ij}}\right)$ and the weights connecting the hidden layer to the output layer $\mathrm{W} 2\left(\mathrm{w}_{\mathrm{jk}}\right)$, which are arranged as a matrix. 
- $\quad$ Choose $\mathrm{X}_{\mathrm{j}}$ as an input and the target output $\mathrm{Y}_{\mathrm{j}}$

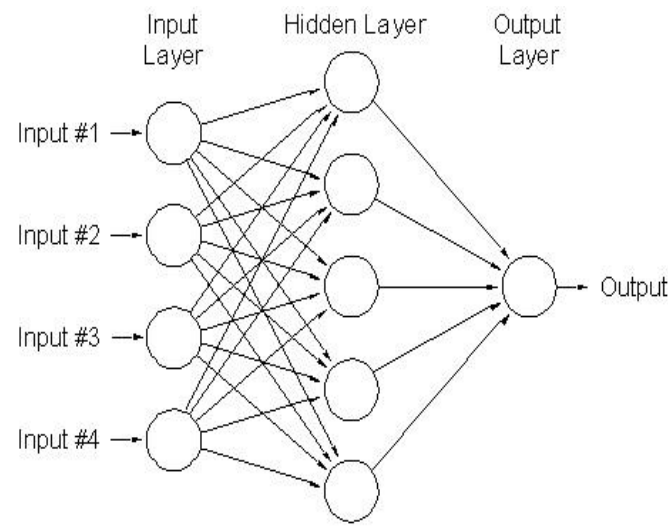

Fig.(2) MLFF Neural Network [3]

\subsection{Back-propagation Learning}

\section{Algorithm}

I) Propagate activation function from input to hidden layer

$$
h i=\frac{1}{1+e^{-\Sigma w 1 i j} x j}
$$

II) Propagate activation function from hidden to the output layer

$$
o j=1 /\left(1+e^{-\sum w 2 j i h i}\right)
$$

III) Calculate the error based on the actual output $o_{j}$ and the target output $y_{j}$

$$
\delta 2 j=o j(1-o j)(y j-o j)
$$

IX) Calculate the error in the hidden layer

$$
\delta 1 i=h i(1-h i) \Sigma \delta 2 j w 2 j i
$$

$\mathrm{X})$ Adjust the weights between hidden layer and output layer

$$
\Delta w 2 j i=\mu \delta 2 j h i
$$

where $\mu=0.5$ (the learning rate)

XI) Adjust the weights between the input layer and hidden layer

$$
\Delta w 1 i j=\mu \delta 1 i x j
$$

XII) Go to step (I) and repeat.

\subsection{Watermarking using Neural Network}

1. Save the weights that are resulted from the algorithm into a file.

2. Enter the noisy image [Fig.(4-a)] into the neural network algorithm with the weights of the resulted filtered image that is shown in Fig(4-b)

3. The resulted image from the neural network will be displayed without noise as is shown in Fig.(4-c).

4. Take the cover image[Fig.(4-d)] and enter it to the neural network algorithm as an input and save the weights into another file.

5. Enter the watermark image [Fig.(4-c)] to the neural network with weights of the covered image then produced the output hiding image that is shown in Fig.(4-e).

6. To extract the cover image from the watermark image enter the produced hiding image to the neural network with the weights of the covered image.

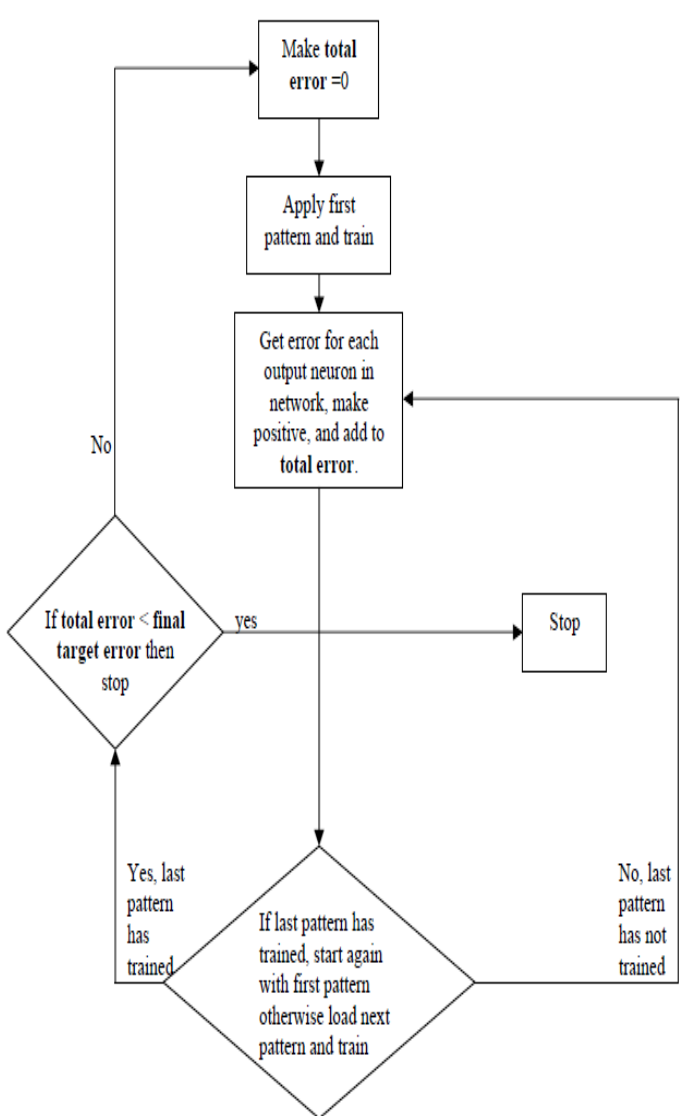

Fig.(3) Back-propagation flowchart 


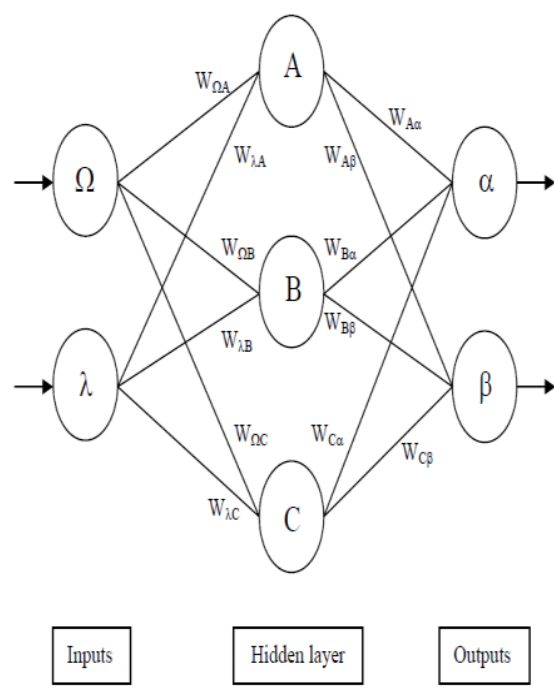

Fig.(4) Weights attached on MLFF NN

\section{SIMULATION RESULTS}

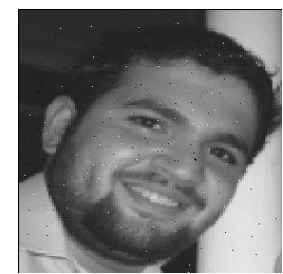

Fig.(4-a) noised image

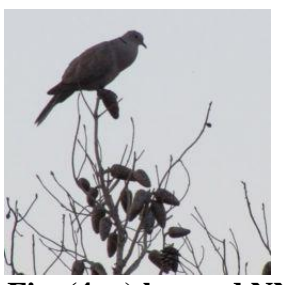

Fig. (4-c ) learned NN image (watermark)

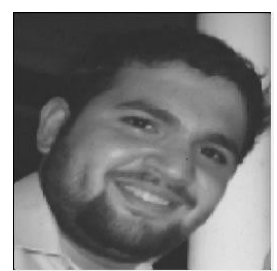

Fig.(4-b) filtered image

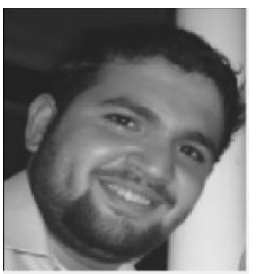

Fig.(4-d) learned NN image with the weights from GF resultant from (4-b) (cover)

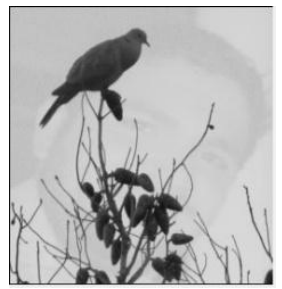

Fig.(4-e) hide image result for watermark(4-c) uploaded with the weights come from cover (4-d).

Fig. (4)- a), b), c), d), e)

Fig.(5)shows the flow chart that represents the whole procedures.

\section{CONCLUSIONS}

This paper describes the use of back-propagation learning algorithm that is applied to the feed-forward multi-layered neural network, with an additional advantage of hiding the trained network weights within the original cover image. The watermarked image has a good robustness and the imperceptibility of the cover image is also highly preserved. For the extraction, only cover image is required and no external weights files need to be supplied with the watermarked image. Thus, this work leads to a successful watermarking scheme. We have outlined an approach to embed a nearly invisible watermark into an image. Although it said to be nearly invisible, it is to find out the watermark visually, for a careful observer. On the other hand, the watermark is unique to individual image, and will be destroy completely in case of small modification, which is a property to against hacking. Finally, since we are using backpropagation learning algorithm to get the weights, these weights are robust enough to feed the feed-forward neural network cover and ordinary image which was processed from noise by the Gaussian filter, and makes it possible to supply only the cover image without any external weight files. By extraction, the weights can be derived from the cover image and used to reconstruct the trained Neural Network again which in turn converts the cover image into desired watermark image.

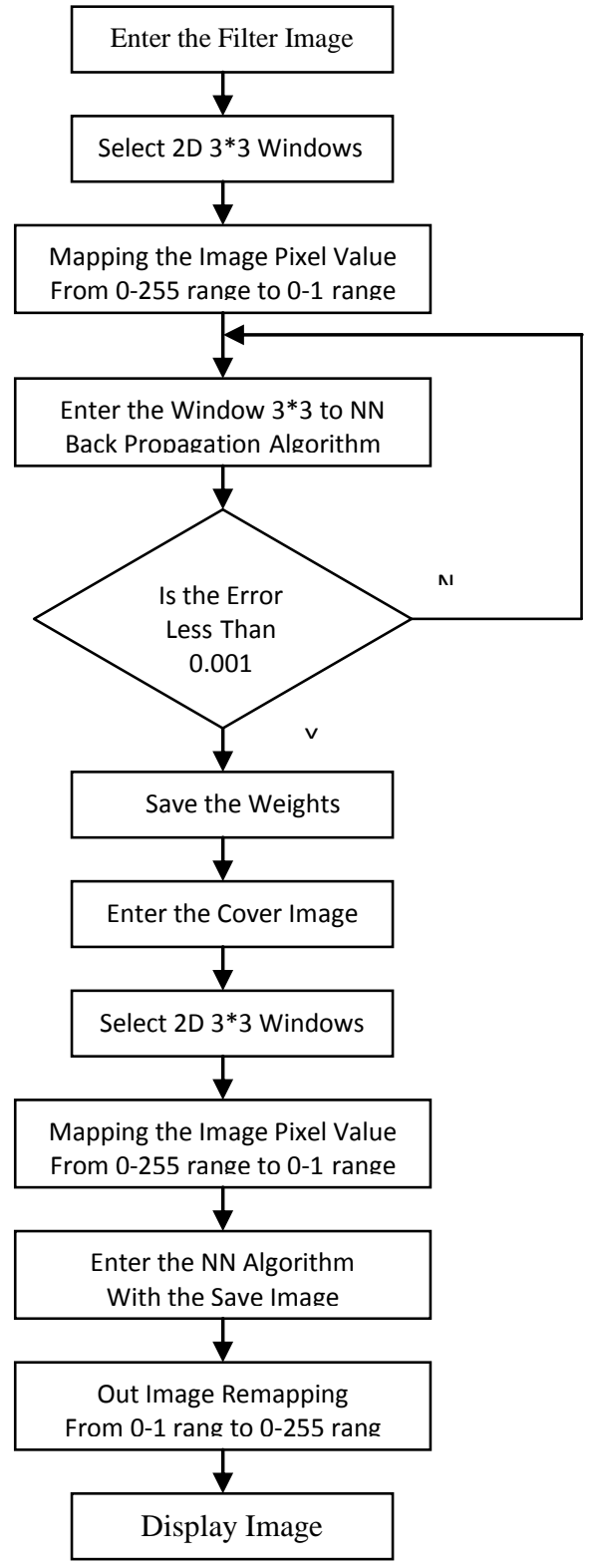

Fig.(5) Flow chart represents the procedure of the proposed approach 


\section{ACKNOWLEDGMENTS}

Our thanks to Referee who read our paper and to the experts who contributed towards development of the template, also our thanks to the Editorial Support Team, International Journal of Computer Applications, Foundation of Computer Science.

\section{REFERENCES}

[1] Fan Z., Hongbin Z.. 2004. CAPACTY AND RELIABILITY OF DIGITAL WATERMARKINGIEEE International Conference on the Business of Electronic Product Reliability and Liability, pp.162- 165,

[2] Cummins J., Diskin P., Lau S. and ParletR. t. 2004. STEGANOGRAPHY AND DIGITAL WATERMARKING http://www.gnu.org/copyleft/fdl.html

[3] Shareef A. Q.. 1995. NEURAL NETWORKS FOR SYSTEM IDENTIFICATION. Ms.c. thesis, University of Technology, Control and System Dep..

[4] Hoffmann G.. 2002. GAUSSIAN FILTER http://www.fho-emden.de/ hoffmann/

[5] Steinebach M., Hauer E., Wolf P.. 2007. EFFICIENT WATERMARKING STRATIGES. Third International Conference on Automated Production of Cross Media Content for Multi-channel Distribution, IEEE: 0-76953030-3/07, pp. 65-71.

[6] Schyndel R. G.van, 1Trkel A. Z.1, Osbome C.F1994. A DIGITAL WATERMARK. IEEE International Conference Image Processing Proceedings ICIP-94, Vol.2, pp. 86-90.

[7] Wong K. K., Tse C. H., K. S. Ng, Lee T. H. and Cheng L.M.1997. ADAPTIVE WATER MARKING.IEEE Transactions on Consumer Electronics, Vol. 43, No. 4, pp.1003-1009.

[8] Joshi A. M., . Darji A, . Mishr V. 2011. DESIGN AND IMPLEMENTATION OF REAL-TIME IMAGEWATERMARKING. IEEE International Conference pp.1-5, 14-16.
[9] Chinnasarn K., Rangsanseri Y., Thitimaishima P.1998. REMOVING SALT-AND-PEPPER NOISE INTEXT/GRAPHICS IMAGES. IEEE Asia-Pacific Conference on APCCAS, Circuits and Systems Journal. pp. $459-462$.

[10] Krishn S.G., Reddy T.S., Rajini G.K.. 2012. REMOVAL OF HIGH DENSITY SALT AND PEPPER NOISE THROUGH MODIFIED DECISION BASED UNSYMMETRIC TRIMMED MEDIANFILTER. International Journal of Engineering Research and Applications (IJERA), Vol. 2, Issue 1, pp.090-094. www.ijera.com.

[11] Chan R.H., Chung-Wa Ho, and Nikolova M. 2004.SALT-AND-PEPPER NOISE REMOVEL BY MEDIAN-TYPE NOISE DETECTORS AND DETAILPRESERVING REGULARIZATION http://www.math.cuhk.edu.hk/ rchan/paper/impulse/imp ulse.pdf.

[12] Yongqiang C., Yanqing Z., and Lihua P.. 2009. A DWT DOMAIN IMAGE WATERMARKING SCHEME USING GENETIC ALGORITHM AND SYNERGETIC NEURAL NETWORK. Proceedings of the International Symposium on Information Processing (ISIP'09) Huangshan, China, pp. 298-301.

[13] Bansal A., Bhadauria S. S.. 2005 - 2008 WATERMARKING USING NEURAL NETWORK AND HIDING THE TRAINED NETWORK WITHIN THE COVER IMAGE. Journal of Theoretical and Applied Information Technology. www.jatit.org

[14] Lai F.H.L, Yong C. Z., Long T.. 2005. NOVEL PERCEPUAL MODELING WATERMARKING WITH MLF NEURAL NETWORKS. International Journal of Information and Communication Engineering, Vol. 1, No.5, pp.266-269.

[15] Oueslati S., Cherif A., Solaimane B.. 2011 ADAPTIVE IMAGE WATERMARKING SCHEME BASED ON NEURAL NETWORK. International Journal of Engineering Science and Technology (IJEST), Vol. 3, No. 1, pp. 748-765. 